\title{
The Wulff shape minimizes an anisotropic Willmore functional
}

\author{
ULRICH CLARENZ ${ }^{\dagger}$ \\ Nordstrasse 13, 53111 Bonn, Germany
}

[Received 2 July 2003]

\begin{abstract}
The aim of this paper is to find a fourth order energy having Wulff shapes as minimizers. This question is motivated by surface restoration problems. In surface restoration usually a damaged region of a surface has to be replaced by a surface patch which restores the region in a suitable way. In particular one aims for $C^{1}$-continuity at the patch boundary. A fourth order energy is considered to measure fairness and to allow appropriate boundary conditions ensuring continuity of the normal field. Here, anisotropy comes into play if edges and corners of a surface are destroyed. In the present paper we define a generalization of the classical Willmore functional and prove that Wulff shapes are its only minimizers.
\end{abstract}

\section{Introduction}

Consider a closed, immersed, oriented, smooth surface $x: \mathcal{M} \rightarrow \mathbb{R}^{3}$ with a two-dimensional parameter manifold $\mathcal{M}$. The differential of the normal mapping $n: \mathcal{M} \rightarrow S^{2}$ induces the shape operator $S$ via $D x \circ S=D n$. The classical Willmore functional is defined as

$$
W[x]=\frac{1}{2} \int_{\mathcal{M}} h^{2} \mathrm{~d} A,
$$

where $\mathrm{d} A$ is the induced area element and $h=\operatorname{tr} S$ is the mean curvature. This functional is used e.g. for modelling elastic surfaces.

A geometric analysis concerning the structure of integrands $f\left(\kappa_{1}, \kappa_{2}\right)$ appearing in elasticity is due to Nitsche. Here, $\kappa_{1}$ and $\kappa_{2}$ are the principal curvatures of $x$. Nitsche considered integrands $f\left(\kappa_{1}, \kappa_{2}\right)$ which are symmetric, definite and of polynomial growth of order at most two. He shows that such integrands are of the form

$$
\alpha+\beta\left(h-h_{0}\right)^{2}-\sigma k
$$

where $\alpha, \beta, \sigma$ and $h_{0}$ are constants fulfilling certain structural inequalities [11]. Furthermore, $k=$ $\operatorname{det} S$ is the classical Gauß curvature. Nevertheless, the physical meaning of the pure Willmore functional ( $\left.\alpha=\sigma=h_{0}=0, \beta=1\right)$ is limited. Any round sphere is a minimizer and the area of Willmore surfaces cannot be bounded.

On the other hand, it is a well known fact that spheres are the only minimizers of $W$. For the construction of minimizers in the classes of fixed genus we refer to [18] in the case of genus one, and to [1] for arbitrary genus.

Recently, the corresponding $L^{2}$-gradient flow of $W$-the Willmore flow-was considered analytically as well as numerically. For initial data close to spheres in the $C^{2, \alpha}$ topology, Simonett

\footnotetext{
†Email: ulrich@clarenz.com
} 
is able to show global existence of the flow and convergence to a sphere [19]. Kuwert and Schätzle [8] prove a lower bound on the maximal time of smooth existence of the Willmore flow in terms of the concentration of the curvature. In [7, 9] they are able to show that for surfaces of sphere type and initial energy less than or equal to $8 \pi$, Willmore flow converges to a round sphere. (Note that in the present paper spheres have energy $8 \pi$ in contrast to $4 \pi$ which is the usual convention in geometry.)

Mayer and Simonett present a numerical scheme for axisymmetric solutions based on finite differences [10]. Their numerical experiments predict the appearance of singularities under Willmore flow in finite time. Furthermore, these experiments show that the result obtained in [9] is optimal.

The case of curves moving in space under Willmore flow (and also curve diffusion) is considered in [5] analytically and numerically. Here, results of Polden for planar curves [13, 14] are generalized and a semi-implicit discretization scheme based on a mixed formulation is given.

A discretization scheme for triangulated surfaces without boundary is obtained by Rusu [15]. A generalization of this to bounded surfaces is used in [2] for applications in surface restoration.

The present work is motivated by restoration problems. Restoring a surface usually means replacing a damaged domain of a surface by a patch which restores the region in a suitable way. This means in particular $C^{1}$-continuity at the boundary [22]. Therefore, it is crucial to use at least fourth order methods if one wants to obtain smoothness at the boundary. With the aid of Willmore flow, one is able to prescribe boundary conditions for the position vector and the normal. Often, realworld restoration problems are of anisotropic nature, e.g., if the edge of a surface is destroyed. In such cases, using the isotropic Willmore functional will not lead to results respecting this anisotropy (see [2]). Therefore, one is interested in anisotropic fourth order functionals with corresponding minimizers. In this paper, we propose to replace the integrand $h^{2}$ by a generalized mean curvature appearing as first variation of the functional

$$
A_{\gamma}[x]=\int_{\mathcal{M}} \gamma(n) \mathrm{d} A .
$$

Here $\gamma$ is a smooth function

$$
\gamma: S^{2} \rightarrow \mathbb{R}^{+}, \quad z \mapsto \gamma(z),
$$

and we may assume that $\gamma$ is given as a one-homogeneous function on $\mathbb{R}^{3}$, i.e., for $\lambda>0$ we have $\gamma(\lambda z)=\lambda \gamma(z)$. In addition, we assume there is a positive constant $m$ such that for the second derivative we have

$$
D^{2}(\gamma(z)-m|z|) \geqslant 0
$$

In this case, $\gamma$ is called elliptic and the eigenvalues of $D^{2} \gamma(z)$ restricted to $z^{\perp}=\left\{x \in \mathbb{R}^{3} \mid x \cdot z=0\right\}$ are bounded from below by $m$. Let us mention here that the Euclidean scalar product of two vectors $a, b \in \mathbb{R}^{3}$ will always be denoted by $a \cdot b$.

Considering a surface $x: \mathcal{M} \rightarrow \mathbb{R}^{3}$, we can give a version of the second derivative of $\gamma$ on its tangential space as follows:

$$
a_{\gamma}: T_{\xi} \mathcal{M} \rightarrow T_{\xi} \mathcal{M}, \quad v \mapsto D x^{-1} \gamma_{z z}(n) D x(v) .
$$

The endomorphism field $a_{\gamma}$ is well defined due to the fact that $D^{2} \gamma(z) z=0$ for all $z \neq 0$. By ellipticity, $a_{\gamma}$ is positive definite. The classical area functional is obtained for the function $\gamma(z)=$ $|z|$. In this case, $a_{\gamma}$ is the identity. 
The first variation of $A_{\gamma}$ in direction $\vartheta$ may be represented in the $L^{2}$-metric by a generalized mean curvature vector:

$$
\left\langle A_{\gamma}^{\prime}[x], \vartheta\right\rangle=\int_{\mathcal{M}} h_{\gamma} n \cdot \vartheta \mathrm{d} A .
$$

Here, $h_{\gamma}=\operatorname{tr}\left(a_{\gamma} S\right)$ will be called the $\gamma$-mean curvature.

It is known since the beginning of the last century [21] that solutions of the isoperimetric problem for $A_{\gamma}$ exist and are given by the so called Wulff shapes $\mathcal{W}_{\gamma}$ that may be obtained from $\gamma$ via the following parametrization over the unit sphere:

$$
\gamma_{z}: S^{2} \rightarrow \mathcal{W}_{\gamma}, \quad z \mapsto \gamma_{z}(z) .
$$

For a proof of the (unique) isoperimetric property of the Wulff shape and more references to the literature see [6].

In this paper we want to give a different characterization of Wulff shapes. For the application in anisotropic restoration problems one seeks for a fourth order functional $W_{\gamma}$ which has the Wulff shape $\mathcal{W}_{\gamma}$ as minimizer. Here we show that one possible choice is

$$
W_{\gamma}[x]=\frac{1}{2} \int_{\mathcal{M}} h_{\gamma}^{2} \mathrm{~d} A .
$$

In this way we have found a fourth order functional which is well suited for anisotropic restoration problems. For a second order approach to surface fairing by locally prescribing Wulff shapes we refer to [3].

The paper is organized as follows: Section 2 contains a proof of a formula for the linearization of a generalized resp. anisotropic mean curvature (Theorem 2.2. This formula may also be interesting in other applications because the anisotropy we consider there is not related to an integrand $\gamma$ as in (2).

In Section 3 we apply the result of Section 2 in the special case of anisotropic mean curvature obtained by an integrand $\gamma$ to derive the Euler equation of the functional (6). An important tool is a generalized Codazzi equation (12). Moreover, we show that the Wulff shape is a solution of the Euler equation.

In Section 4 we prove the main result of this paper. Here, it is shown that Wulff shapes are not only stationary points of $W_{\gamma}$ but also minimizers and essentially the only minimizers. The proof is based on a symmetrization argument (16) and the generalized Codazzi equation (12). For a related reasoning see the work on stable surfaces of constant $\gamma$-mean curvature [12].

\section{Linearization of generalized mean curvature}

In this section we will consider a family of surfaces $x: \mathcal{M} \times(-\eta, \eta) \rightarrow \mathbb{R}^{3}$, thus $x(t)=x(\cdot, t)$ is an immersion of a two-dimensional orientable manifold $\mathcal{M}$ and $\eta$ is a small positive real number. This family is considered as a perturbation of a surface $x: \mathcal{M} \rightarrow \mathbb{R}^{3}$, i.e., $x(\cdot, 0)=x$. The evolution of $x(t)$ is assumed to be given by the equation

$$
\partial_{t} x=\varphi(t) n(t)+D x(v(t)),
$$

where $\varphi(t)$ is a smooth function and $v(t)$ is a smooth vector field on $\mathcal{M}$.

The following notion is essential in our considerations: 
DEFINITION 2.1 Let $\alpha \in \mathbb{R}^{3 \times 3}$ be a symmetric endomorphism depending on $z \in S^{2}$ with the property $\alpha(z) z=0$ for all $z \in S^{2}$. Then $\alpha$ induces an endomorphism field on $\mathcal{M}$,

$$
\bar{a}: \mathcal{T} \mathcal{M} \rightarrow \mathcal{T} \mathcal{M}
$$

given by $\bar{a}=D x^{-1} \circ \alpha \circ D x$. The corresponding generalized mean curvature is defined as

$$
h_{\bar{a}}=\operatorname{tr}(\bar{a} \circ S) .
$$

In the next section we will apply this notion in the case of $\alpha(z)=\gamma_{z z}(z)$ for an elliptic integrand $\gamma$ as in (2). In this case we assume that $\alpha(z): z^{\perp} \rightarrow z^{\perp}$ is positive definite for all $z \in S^{2}$. The main result of this section will be

THEOREM 2.2 Let $x$ be a family of surfaces evolving according to (7). The generalized mean curvature $h_{\bar{a}}$ fulfils the following evolution equation:

$$
-\partial_{t} h_{\bar{a}}=\Delta_{\bar{a}} \varphi+|S|_{\bar{a}}^{2} \varphi-g(\operatorname{div} \bar{a}, \operatorname{grad} \varphi)-\operatorname{tr}\left(\bar{a} \circ\left[\left(\nabla_{\bullet} S\right) v\right]\right)-g^{i j}\left(\partial_{t} \alpha \partial_{i} x\right) \cdot \partial_{j} n .
$$

Here we use $\Delta_{\bar{a}}=\operatorname{div}(\bar{a} \operatorname{grad})$ and $|S|_{\bar{a}}^{2}=\operatorname{tr}\left(\bar{a} \circ S^{2}\right)$.

Proof. Equation (7) implies the following equation for the normal $n$ :

$$
\partial_{t} n=-D x(\operatorname{grad} \varphi)+D n(v) .
$$

With the classical notation for $g_{i j}, g^{i j}$ and $h_{\bar{a}, i j}=-g\left(\bar{a} \partial_{i}, S \partial_{j}\right)=-\left(\alpha \partial_{i} x\right) \cdot \partial_{j} n$, the mean curvature $h_{\bar{a}}$ can be written as

$$
-h_{\bar{a}}=g^{i j} h_{\bar{a}, i j}
$$

and consequently $-\partial_{t} h_{\bar{a}}=\partial_{t} g^{i j} h_{\bar{a}, i j}+g^{i j} \partial_{t} h_{\bar{a}, i j}=I+I I$. Here and in the following we will use the Einstein summation convention. Since

$$
\partial_{t} g^{i j}=-g^{i k} \partial_{t} g_{k l} g^{l j}
$$

we can state $\left(h_{i j}=-\partial_{i} x \cdot \partial_{j} n\right)$

$$
\partial_{t} g^{i j}=2 \varphi g^{i k} h_{k l} g^{l j}-g^{i k} D x\left(\nabla_{k} v\right) \cdot \partial_{l} x g^{l j}-g^{i k} \partial_{k} x \cdot D x\left(\nabla_{l} v\right) g^{l j} .
$$

Note that for the tangential part of $\partial_{k} D x(v)$ we have $\left[\partial_{k} D x(v)\right]^{\tan }=D x\left(\nabla_{k} v\right)$. Thus for the term $I$ we obtain

$$
\begin{aligned}
I & =2 \varphi g^{i k} h_{k l} g^{l j} h_{\bar{a}, i j}-g^{i k} D x\left(\nabla_{k} v\right) \cdot \partial_{l} x g^{l j} h_{\bar{a}, i j}-g^{i k} \partial_{k} x \cdot D x\left(\nabla_{l} v\right) g^{l j} h_{\bar{a}, i j} \\
& =2 \varphi|S|_{\bar{a}}^{2}+g^{i k} g\left(S \bar{a} \partial_{i}, \nabla_{k} v\right)+g^{l j} g\left(\nabla_{l} v, \bar{a} S \partial_{j}\right)=2 \varphi|S|_{\bar{a}}^{2}+\operatorname{tr}\left((\bar{a} S+S \bar{a}) \nabla_{\bullet} v\right) .
\end{aligned}
$$

The computation of $\partial_{t} h_{\bar{a}, i j}$ gives

$$
\begin{aligned}
\partial_{t} h_{\bar{a}, i j}= & -\partial_{t} \alpha \partial_{i} x \cdot \partial_{j} n-\alpha \partial_{i}(\varphi n+D x(v)) \cdot \partial_{j} n-\alpha \partial_{i} x \cdot \partial_{j}(-D x(\operatorname{grad} \varphi)+D n(v)) \\
= & -\partial_{t} \alpha \partial_{i} x \cdot \partial_{j} n-\varphi \alpha \partial_{i} n \cdot \partial_{j} n-\alpha D x\left(\nabla_{i} v\right) \cdot \partial_{j} n \\
& +\alpha \partial_{i} x \cdot \partial_{j} D x(\operatorname{grad} \varphi)-\alpha \partial_{i} x \cdot \partial_{j} \operatorname{Dn}(v) \\
= & -\partial_{t} \alpha \partial_{i} x \cdot \partial_{j} n-\varphi \alpha \partial_{i} n \cdot \partial_{j} n-\alpha D x\left(\nabla_{i} v\right) \cdot \partial_{j} n \\
& +\alpha \partial_{i} x \cdot \partial_{j} D x(\operatorname{grad} \varphi)-\alpha \partial_{i} x \cdot D x\left(S \nabla_{j} v\right)-\alpha \partial_{i} x \cdot D x\left(\left(\nabla_{j} S\right) v\right) .
\end{aligned}
$$


Therefore, the term II can be geometrically interpreted as follows:

$$
\begin{aligned}
I I= & -g^{i j} \partial_{t} \alpha \partial_{i} x \cdot \partial_{j} n-\varphi g^{i j} \alpha \partial_{i} n \cdot \partial_{j} n-g^{i j} \alpha D x\left(\nabla_{i} v\right) \cdot \partial_{j} n \\
& +g^{i j} \alpha \partial_{i} x \cdot \partial_{j} D x(\operatorname{grad} \varphi)-g^{i j} \alpha \partial_{i} x \cdot D x\left(S \nabla_{j} v\right)-g^{i j} \alpha \partial_{i} x \cdot D x\left(\left(\nabla_{j} S\right) v\right) \\
= & -g^{i j} \partial_{t} \alpha \partial_{i} x \cdot \partial_{j} n-\varphi|S|_{\bar{a}}^{2}-\operatorname{tr}\left(S \bar{a} \nabla_{\bullet} v\right) \\
& +g^{i j} \alpha \partial_{i} x \cdot \partial_{j} D x(\operatorname{grad} \varphi)-\operatorname{tr}\left(\bar{a} S \nabla_{\bullet} v\right)-\operatorname{tr}\left(\bar{a} \circ\left[\left(\nabla_{\bullet} S\right) v\right]\right) .
\end{aligned}
$$

Now, we finish the proof. The linearization of $h_{\bar{a}}$ is the sum of $I$ and $I I$ :

$$
\begin{aligned}
-\partial_{t} h_{\bar{a}}= & \varphi|S|_{\bar{a}}^{2}+g^{i j} \partial_{i} x \cdot \alpha \partial_{j} D x(\operatorname{grad} \varphi)-g^{i j} \partial_{t} \alpha \partial_{i} x \cdot \partial_{j} n-\operatorname{tr}(\bar{a} \circ[(\nabla \cdot S) v]) \\
= & \varphi|S|_{\bar{a}}^{2}+g^{i j} \partial_{i} x \cdot \partial_{j}(\alpha D x(\operatorname{grad} \varphi))-g^{i j} \partial_{i} x \cdot \partial_{j} \alpha D x(\operatorname{grad} \varphi) \\
& -g^{i j} \partial_{t} \alpha \partial_{i} x \cdot \partial_{j} n-\operatorname{tr}(\bar{a} \circ[(\nabla \cdot S) v]) \\
= & \Delta_{\bar{a}} \varphi+|S|_{\bar{a}}^{2} \varphi-g^{i j} \partial_{i} x \cdot \partial_{j} \alpha D x(\operatorname{grad} \varphi)-\operatorname{tr}(\bar{a} \circ[(\nabla \cdot S) v])-g^{i j} \partial_{t} \alpha \partial_{i} x \cdot \partial_{j} n .
\end{aligned}
$$

It remains to show $g(\operatorname{div} \bar{a}, w)=g^{i j} \partial_{i} \alpha \partial_{j} x \cdot D x(w)$ for all vector fields $w$ :

$$
\begin{aligned}
g(\operatorname{div} \bar{a}, w) & =g^{i j} g\left(\nabla_{i} \bar{a} \partial_{j}, w\right)=g^{i j} D x\left(\left(\nabla_{i} \bar{a}\right) \partial_{j}\right) \cdot D x(w) \\
& =g^{i j} \operatorname{Dx}\left(\nabla_{i}\left(\bar{a} \partial_{j}\right)-\bar{a} \nabla_{i} \partial_{j}\right) \cdot D x(w) \\
& =g^{i j} \partial_{i} D x\left(\bar{a} \partial_{j}\right)-\alpha D x\left(\nabla_{i} \partial_{j}\right) \cdot D x(w)=g^{i j} \partial_{i} \alpha \partial_{j} x \cdot D x(w) .
\end{aligned}
$$

\section{Anisotropic Willmore energies}

Now we want to apply the result on the linearization of generalized mean curvature to compute the derivative of anisotropic Willmore functionals.

We consider the energy $W_{\gamma}$ defined as in $\sqrt[6]{6}$. For a test function $\vartheta \in C^{1}\left(\mathcal{M}, \mathbb{R}^{3}\right)$ we have

$$
\left\langle W_{\gamma}^{\prime}[x], \vartheta\right\rangle=\frac{\mathrm{d}}{\mathrm{d} t} W_{\gamma}\left[x_{t}\right]_{\mid t=0},
$$

where $x_{t}$ fulfils $\partial_{t} x_{t \mid t=0}=\vartheta$. According to Section 2 we split $\vartheta$ into a normal component $\varphi n$ and a tangential component $D x(v)$, i.e., $\vartheta=\varphi n+D x(v)$.

To derive the Euler equation we have to compute the derivative of $h_{\gamma, t}^{2}$ and of the area element $\mathrm{d} A_{t}$. The latter is contained in [17] and the result is $\partial_{t} \mathrm{~d} A_{t \mid t=0}=\operatorname{div} \vartheta \mathrm{d} A$.

In the special case where the endomorphism field $\bar{a}$ from Section 2 is given by the second derivative of an elliptic integrand $\gamma$, Theorem 2.2 simplifies in the following way:

By (11) for $\alpha=\gamma_{z z}$ we have, due to $\partial_{t} n_{t \mid t=0}=-D x(\operatorname{grad} \varphi)+D n(v)$,

$$
\begin{aligned}
g^{i j}\left(\partial_{t} \alpha_{t \mid t=0} \partial_{i} x\right) \cdot \partial_{j} n & =g^{i j} \gamma_{z z z}(n)\left[\partial_{t} n_{t \mid t=0}, \partial_{i} x, \partial_{j} n\right] \\
& =g^{i j} \partial_{j}\left(\gamma_{z z}(n)\right)(-D x(\operatorname{grad} \varphi)+D n(v)) \cdot \partial_{i} x \\
& =g\left(\operatorname{div} a_{\gamma},-\operatorname{grad} \varphi+S v\right),
\end{aligned}
$$

where $a_{\gamma}$ is defined as in (3). For the linearization of $h_{\gamma}:=h_{a_{\gamma}}$ we therefore obtain, with $\Delta_{\gamma}=$ $\operatorname{div}\left(a_{\gamma} \operatorname{grad}\right)$ and $|S|_{\gamma}^{2}=\operatorname{tr}\left(a_{\gamma} S^{2}\right)$,

$$
-\partial_{t} h_{\gamma}=\Delta_{\gamma} \varphi+|S|_{\gamma}^{2} \varphi-\operatorname{tr}\left(a_{\gamma} \circ[(\nabla \cdot S) v]\right)-g\left(\operatorname{div} a_{\gamma}, S v\right) .
$$


The Codazzi equation as well as the symmetry of $S$ and $a_{\gamma}$ imply the following

LEMMA 3.1 For the divergence of the endomorphism field $S a_{\gamma}$ and all vector fields $v$ the identity

$$
g\left(\operatorname{div}\left(S a_{\gamma}\right), v\right)=\operatorname{tr}\left(a_{\gamma} \circ\left[\left(\nabla_{\bullet} S\right) v\right]\right)+g\left(\operatorname{div} a_{\gamma}, S v\right)
$$

is valid. Furthermore, for $\varphi=0$ in (7) the tangential part of $\partial_{t} h_{\gamma}$ is $g\left(\operatorname{grad} h_{\gamma}, v\right)$ from which one concludes

$$
\operatorname{div}\left(S a_{\gamma}\right)=\operatorname{grad} h_{\gamma} .
$$

Proof. By definition of the divergence, one gets

$$
\begin{aligned}
g\left(\operatorname{div}\left(S a_{\gamma}\right), v\right) & =g^{i k} g\left(\nabla_{i}\left(S a_{\gamma}\right) \partial_{k}, v\right)=g^{i k} g\left(\left(\nabla_{i} S\right) a_{\gamma} \partial_{k}, v\right)+g^{i k} g\left(S\left(\nabla_{i} a_{\gamma}\right) \partial_{k}, v\right) \\
& =g^{i k} g\left(a_{\gamma} \partial_{k},\left(\nabla_{i} S\right) v\right)+g^{i k} g\left(\left(\nabla_{i} a_{\gamma}\right) \partial_{k}, S v\right) \\
& =g^{i k} g\left(\partial_{i},\left(\nabla_{a_{\gamma}} \partial_{k} S\right) v\right)+g\left(\operatorname{div} a_{\gamma}, S v\right)=\operatorname{tr}\left(\left(\nabla_{a_{\gamma}} \cdot S\right) v\right)+g\left(\operatorname{div} a_{\gamma}, S v\right)
\end{aligned}
$$

Defining the endomorphism $\Sigma$ via $\Sigma w=\left(\nabla_{w} S\right) v$ we have

$$
\operatorname{tr}\left(\left(\nabla_{a_{\gamma} \bullet} S\right) v\right)=\operatorname{tr}\left(\Sigma \circ a_{\gamma}\right)=\operatorname{tr}\left(a_{\gamma} \circ \Sigma\right)
$$

and the result is shown.

\section{The Euler equation of $W_{\gamma}$}

From the above considerations we obtain the identity

$$
-\partial_{t} h_{\gamma}=\Delta_{\gamma} \varphi+|S|_{\gamma}^{2} \varphi-g\left(\operatorname{grad} h_{\gamma}, v\right) .
$$

Using this result on the linearization of $\gamma$-mean curvature, we obtain

$$
\begin{aligned}
\left\langle W_{\gamma}^{\prime}[x], \vartheta\right\rangle & =\int_{\mathcal{M}}\left(h_{\gamma}\left(-\Delta_{\gamma} \varphi-|S|_{\gamma}^{2} \varphi+g\left(\operatorname{grad} h_{\gamma}, v\right)\right)+\frac{1}{2} h_{\gamma}^{2} \operatorname{div} \vartheta\right) \mathrm{d} A \\
& =\int_{\mathcal{M}}\left(h_{\gamma}\left(-\Delta_{\gamma} \varphi-|S|_{\gamma}^{2} \varphi\right)+\frac{1}{2} h h_{\gamma}^{2} \varphi+\frac{1}{2} \operatorname{div}\left(h_{\gamma}^{2} v\right)\right) \mathrm{d} A .
\end{aligned}
$$

This relation implies the Euler equation of the anisotropic Willmore functional for surfaces without boundary:

$$
-\Delta_{\gamma} h_{\gamma}-h_{\gamma}|S|_{\gamma}^{2}+\frac{1}{2} h h_{\gamma}^{2}=0
$$

In particular we obtain the following

PROPOSITION 3.2 The Wulff shape $\mathcal{W}_{\gamma}$ is a solution of the Euler equation for the anisotropic Willmore functional.

Proof. We use the parametrization of the Wulff shape given in 5 . The normal of $\mathcal{W}_{\gamma}$ at $D \gamma(z)$ is given by $z$ because $D^{2} \gamma(z) z=0$ for $z \neq 0$. Therefore, one gets $S=a_{\gamma}^{-1}$ for the shape operator. This in particular implies that on the Wulff shape we have $h_{\gamma}=$ const. The result will be shown if we can prove

$$
|S|_{\gamma}^{2}-\frac{1}{2} h_{\gamma} h=0
$$


but this is a consequence of the above considerations:

$$
\begin{aligned}
|S|_{\gamma}^{2} & =\operatorname{tr}\left(a_{\gamma} S^{2}\right)=\operatorname{tr} a_{\gamma}^{-1}, \\
\frac{1}{2} h_{\gamma} h & =\frac{1}{2} \operatorname{tr}(\mathrm{Id}) \operatorname{tr} a_{\gamma}^{-1}=\operatorname{tr} a_{\gamma}^{-1} .
\end{aligned}
$$

This proposition clearly follows from the result of the next section. Nevertheless, the discussion of the Euler equation seems worth recording.

\section{Main result}

As was pointed out in the introduction, spheres are not only extremals but also minimizers of the classical Willmore functional. The aim of this section is to prove an anisotropic version of this result. We will show

THEOREM 4.1 Let $x: \mathcal{M} \rightarrow \mathbb{R}^{3}$ be an immersion of a compact surface $\mathcal{M}$ without boundary into $\mathbb{R}^{3}$. We can estimate the anisotropic Willmore energy $W_{\gamma}[x]$ from below by

$$
W_{\gamma}[x] \geqslant 2\left|\mathcal{W}_{\gamma}\right|,
$$

where $\left|\mathcal{W}_{\gamma}\right|$ is the area of the Wulff shape $\mathcal{W}_{\gamma}$. The Wulff shape itself is the unique minimizer of $W_{\gamma}$.

Proof. We follow the classical proof given e.g. in [20, pp. 270]. First we want to estimate $W_{\gamma}$ by a total curvature term. In the case of elliptic integrands, the $\gamma$-mean curvature $h_{\gamma}$ may also be written as

$$
h_{\gamma}=\operatorname{tr}\left(a_{\gamma} S\right)=\operatorname{tr}\left(a_{\gamma}^{1 / 2} S a_{\gamma}^{1 / 2}\right) .
$$

The endomorphism field $a_{\gamma}^{1 / 2} S a_{\gamma}^{1 / 2}$ is symmetric and may be diagonalized with eigenvalues $\mu_{1}, \mu_{2}$. Related symmetrizations were also used in [16] and [4]. Introducing the corresponding anisotropic Gauß curvature $k_{\gamma}=\operatorname{det}\left(a_{\gamma} S\right)=\operatorname{det}\left(a_{\gamma}^{1 / 2} S a_{\gamma}^{1 / 2}\right)$, one obtains the relation

$$
h_{\gamma}^{2}-4 k_{\gamma}=\left(\mu_{1}+\mu_{2}\right)^{2}-4 \mu_{1} \mu_{2}=\left(\mu_{1}-\mu_{2}\right)^{2} \geqslant 0
$$

and therefore we can give the following estimate:

$$
W_{\gamma}[x] \geqslant \frac{1}{2} \int_{k_{\gamma}^{+}} h_{\gamma}^{2} \mathrm{~d} A \geqslant 2 \int_{k_{\gamma}^{+}} k_{\gamma} \mathrm{d} A,
$$

where $k_{\gamma}^{+}=\left\{\xi \in \mathcal{M} \mid k_{\gamma}(\xi) \geqslant 0\right\}$. By the area formula, the expression $\int_{k_{\gamma}^{+}} k_{\gamma} \mathrm{d} A$ is the area (counted with multiplicity) of $D \gamma\left(n\left(k_{\gamma}^{+}\right)\right)$. Due to the ellipticity of $\gamma$, we conclude that

$$
k_{\gamma}^{+}=\{\xi \in \mathcal{M} \mid k(\xi) \geqslant 0\},
$$

where $k$ is the classical Gauß curvature. Therefore, and on account of the compactness of $\mathcal{M}$, we obtain $n\left(k_{\gamma}^{+}\right)=S^{2}$ and in particular

$$
\int_{k_{\gamma}^{+}} k_{\gamma} \mathrm{d} A \geqslant\left|\mathcal{W}_{\gamma}\right| .
$$


Assume now that equality holds in all of the above inequalities. Then we conclude that $\mu_{1}=\mu_{2}=$ : $\mu$. Thus we have $a_{\gamma}^{1 / 2} S a_{\gamma}^{1 / 2}=\mu \mathrm{Id}$, from which we get $S a_{\gamma}=\mu \mathrm{Id}$ and therefore

$$
\operatorname{div}\left(S a_{\gamma}\right)=\operatorname{grad} \mu \text {. }
$$

On the other hand we know by Lemma 3.1 (see $(12)$ ) that

$$
\operatorname{div}\left(S a_{\gamma}\right)=\operatorname{grad} h_{\gamma}
$$

This implies $h_{\gamma}-\mu=$ const. Since $h_{\gamma}=\operatorname{tr}\left(S a_{\gamma}\right)=2 \mu$ one gets $\mu=$ const. Thus we obtain

$$
D x\left(a_{\gamma} S-\mu \mathrm{Id}\right)=D \gamma_{z}(n)-\mu D x=0
$$

and integration leads to

$$
x=x_{0}+\frac{1}{\mu} \gamma_{z}(n),
$$

where $x_{0} \in \mathbb{R}^{3}$ is a constant vector, and the result is shown.

\section{Acknowledgements}

The author enjoyed the hospitality of the Mathematische Institut at the University of Freiburg in September 2002 and March 2003. He would like to thank Prof. Dr. Gerhard Dziuk for offering the possibility to be in Freiburg and Prof. Dr. Ernst Kuwert for discussing the problem of minimizers of anisotropic Willmore energies.

\section{REFERENCES}

1. Bauer, M. \& Kuwert, E. Existence of minimizing Willmore surfaces of prescribed genus. Int. Math. Res. Not. 2003, no. 10, 553-576. Zbl 1029.53073 MR 1941840

2. Clarenz, U., Diewald, U., Dziuk, G., Rumpf, M., \& Rusu, R. A finite element method for surface restoration with smooth boundary conditions. Comput. Aided Geom. Design 21 (2004), 427-445. MR 2058390

3. Clarenz, U., Dziuk, G., \& RumpF, M. On generalized mean curvature flow. Geometric Analysis and Nonlinear Partial Differential Equations, H. Karcher and S. Hildebrandt (eds.), Springer (2003), 217-248. Zbl 1035.53091 MR 2008341

4. Clarenz, U. \& VON Der Mosel, H. Compactness theorems and an isoperimetric inequality for critical points of elliptic parametric functionals. Calc. Var. 12 (2000), 85-107. Zbl 0968.35039 MR 1808108

5. Dziuk, G., Kuwert, E., \& Schätzle, R. Evolution of elastic curves in $\mathbb{R}^{n}$ : existence and computation. SIAM J. Math. Anal. 33 (2002), 1228-1245. Zbl 1031.53092 MR 1897710

6. Fonseca, I. \& MÜller, S. A uniqueness proof for the Wulff theorem. Proc. Roy. Soc. Edinburgh Sect. A 119 (1991), 125-136. Zbl 0752.49019 MR 1130601

7. Kuwert, E. \& Schätzle, R. The Willmore flow with small initial energy. J. Differential Geom. 57 (2001), 409-441. Zbl 1035.53092 MR 1882663

8. Kuwert, E. \& Schätzle, R. Gradient flow for the Willmore functional. Comm. Anal. Geom. 10 (2002), 307-339. Zbl 1029.53082 MR 1900754

9. KUWERT, E. \& SCHÄTZLE, R. Removability of point singularities of Willmore surfaces. Preprint SFB 611, Bonn, 47 (2002). 
10. Mayer, U. F. \& Simonett, G. A numerical scheme for axisymmetric solutions of curvature driven free boundary problems with applications to the Willmore flow. Interfaces Free Bound. 4 (2002), 89-109. Zbl 1005.65095 MR 1877537

11. Nitsche, J. C. C. Periodic surfaces that are extremal for energy functionals containing curvature functions. Statistical Thermodynamics and Differential Geometry of Microstructured Materials, H. T. Davis and J. C. C. Nitsche (eds.), Springer (1993), 69-98. Zbl 0794.53007 MR 1226921

12. Palmer, B. Stability of the Wulff shape. Proc. Amer. Math. Soc. 126 (1998), 3661-3667. Zbl 0924.53009 MR 1473676

13. Polden, A. Closed curves of least total curvature. SFB 382 Tübingen, Preprint, 13 (1995).

14. Polden, A. Curves and surfaces of least total curvature and fourth-order flows. Dissertation, Universität Tübingen (1996).

15. RUSU, R. An algorithm for the elastic flow of surfaces. Preprint Mathematische Fakultät Freiburg, 01-35 (2002).

16. SaUvigny, F. Curvature estimates for immersions of minimal surface type via uniformization and theorems of Bernstein type. Manuscripta Math. 67 (1990), 69-97. Zbl 0703.53050 MR 1037997

17. Simon, L. Lectures on Geometric Measure Theory. Proc. Centre Math. Anal. Austral. Nat. Univ. 3 (1984). Zbl 0546.49019 MR 0756417

18. Simon, L. Existence of surfaces minimizing the Willmore functional. Comm. Anal. Geom. 1 (1993), 281-326. Zbl 0848.58012 MR 1243525

19. Simonett, G. The Willmore flow near spheres. Differential Integral Equations 14 (2001), 1005-1014. Zbl pre01832854 MR 1827100

20. Willmore, T. J. Riemannian Geometry. Clarendon Press, Oxford (1993). Zbl 0797.53002 MR 1261641

21. WulfF, G. Zur Frage der Geschwindigkeit des Wachsthums und der Auflösung der Kristallflächen. Z. Kristallographie 34 (1901), 449-530.

22. Yoshizawa, S. \& Belyaev, A. G. Fair triangle mesh generation with discrete elastica. Geometric Modeling and Processing, RIKEN, Saitama (2002), 119-123. 